\title{
Kinematics and Dynamics Are Not Represented Independently in Motor Working Memory: Evidence from an Interference Study
}

\author{
Christine Tong, ${ }^{1}$ Daniel M. Wolpert, ${ }^{2}$ and J. Randall Flanagan ${ }^{1}$ \\ ${ }^{1}$ Department of Psychology and Centre for Neuroscience Studies, Queen's University, Kingston, Ontario, K7L 3N6, \\ Canada, and 2Sobell Department of Neurophysiology, Institute of Neurology, University College London, London, \\ WC1N 3BG, United Kingdom
}

\begin{abstract}
Our capacity to learn multiple dynamic and visuomotor tasks is limited by the time between the presentations of the tasks. When subjects are required to adapt to equal and opposite position-dependent visuomotor rotations (Krakauer et al., 1999) or velocity-dependent force fields (Brashers-Krug et al., 1996) in quick succession, interference occurs that prevents the first task from being consolidated in memory. In contrast, such interference is not observed between learning a positiondependent visuomotor rotation and an acceleration-dependent force field. On the basis of this finding, it has been argued that internal models of kinematic and dynamic sensorimotor transformations are learned independently (Krakauer et al., 1999). However, these findings are also consistent with the perturbations interfering only if they depend on the same kinematic variable. We evaluated this hypothesis using kinematic and dynamic transformations matched in terms of the kinematic
\end{abstract}

The problem of motor learning is one of mastering novel sensorimotor transformations that relate motor commands to sensory outcomes. Such learning involves the acquisition of internal models that capture these sensorimotor transformations and enable the CNS to accurately estimate the motor commands required to achieve desired outcomes and to predict the consequences of actions (Johansson and Cole, 1992; Miall et al., 1993; Shadmehr and Mussa-Ivaldi, 1994; Wolpert et al., 1995; Conditt et al., 1997; Flanagan and Wing, 1997; Kawato, 1999; Wolpert and Ghahramani, 2000). Two classes of sensorimotor transformations have been widely used in motor control research: kinematic and dynamic. Kinematic transformations are mappings between different geometric variables (and their derivatives) and, importantly, do not depend on the dynamic properties of the system. In contrast, dynamic transformations relate motor commands to the motion of the system; therefore, they do depend on dynamic properties such as inertia and viscosity. Thus, for example, to control a computer mouse, we must learn the kinematic transformation that relates mouse motion to cursor motion and the dynamic transformation that relates the forces applied to the mouse to its resultant motion, a mapping that will depend on the inertia of the mouse and the friction between the mouse and the mouse pad.

\footnotetext{
Received Aug. 27, 2001; revised Oct. 31, 2001; accepted Nov. 12, 2001.

This work was supported by the Natural Sciences and Engineering Research Council of Canada, the Wellcome Trust, and the Human Frontier Science Program.

Correspondence should be addressed to J. Randall Flanagan, Department of Psychology, Queen's University, Kingston, ON K7L 3N6, Canada. E-mail: flanagan@psyc.queensu.ca.

Copyright (C) 2002 Society for Neuroscience $\quad 0270-6474 / 02 / 221108-06 \$ 15.00 / 0$
}

variable on which they depend. Subjects adapted to a positiondependent visuomotor rotation followed $5 \mathrm{~min}$ later by a position-dependent rotary force field either with or without visual feedback of arm position. The force field tended to rotate the hand in the direction opposite to the visuomotor rotation. To assess learning, all subjects were retested $24 \mathrm{hr}$ later on the visuomotor rotation, and their performance was compared with a control group exposed only to the visuomotor rotation on both days. Adapting to the position-dependent force field, both with and without visual feedback, impaired learning of the visuomotor rotation. Thus, interference between our kinematic and dynamic transformations was observed, suggesting that the key determinant of interference is the kinematic variable on which the transformation depends.

Key words: motor learning; internal models; arm movement; visuomotor rotation; force field; motor memory

Brashers-Krug et al. (1996) have shown that learning internal models of novel dynamics involves a period of consolidation, during which motor memory is susceptible to disruption. If people adapt to two equal and opposite viscous force fields in quick succession, performance on the second is impaired (anterograde interference) and the memory of the first is overwritten (retrograde interference). Similar interference effects are observed when learning opposing visuomotor rotations (Krakauer et al., 1999; Wigmore et al., 2001).

Although opposing kinematic and opposing dynamic transformations interfere with each other, Krakauer et al. (1999) observed independent learning of internal models for novel kinematic and dynamic transformations. They showed that learning a visuomotor rotation is not affected by adaptation to an inertial load presented either simultaneously or 5 min later. Similarly, a recent study shows lack of anterograde interference between a visuomotor rotation and a viscous force field (Flanagan et al., 1999).

In summary, these studies of motor interference have shown interference between two position-dependent visuomotor mappings and interference between two velocity-dependent force fields but no interference between a position-dependent visuomotor rotation and either an acceleration-dependent or velocitydependent force field. Based on these results, Krakauer et al. (1999) concluded that internal models of kinematic and dynamic transformations are stored in distinct systems of working memory. An alternative hypothesis is that transformations that depend on different kinematic parameters do not interfere, but those that depend on similar kinematic parameters do interfere. 


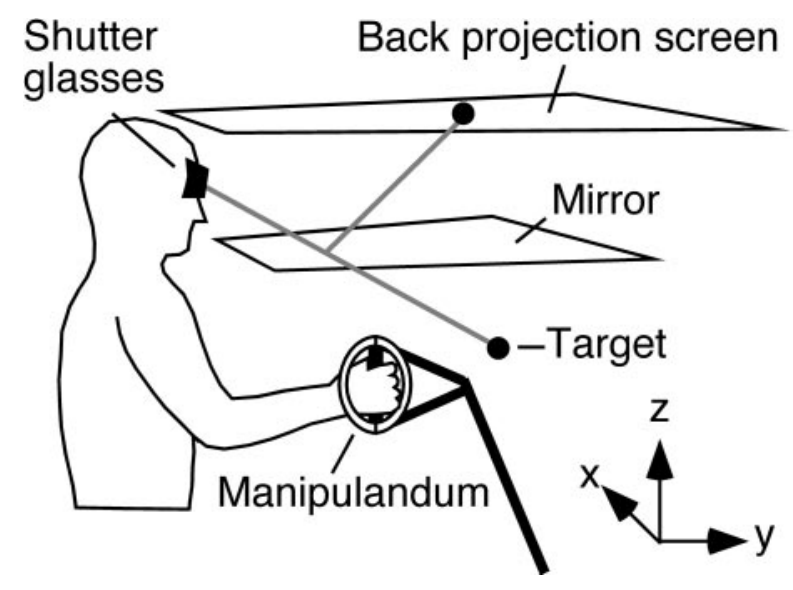

Figure 1. Experimental setup. Subjects moved a force-reflecting manipulandum between targets in a horizontal plane. The targets were virtual spheres presented using a three-dimensional projection system with shutter glasses. The force exerted by the manipulandum was servo-controlled to create a position-dependent rotary force field, and visual feedback was altered to create a position-dependent visuomotor rotation.

We tested this hypothesis using kinematic and dynamic transformations matched by the kinematic parameter on which they depend. In particular, we used a position-dependent visuomotor rotation and a position-dependent rotary force field. The hypothesis that kinematic and dynamic learning are independent predicts that adapting to the force field soon after adapting to the visuomotor rotation should not interfere with consolidation of the former. The hypothesis that transformations that depend on the same kinematic parameter interfere with one another predicts the opposite finding (i.e., that dynamic learning should interfere with the consolidation of kinematic learning).

\section{MATERIALS AND METHODS}

Subjects. After providing written informed consent, 26 subjects (15 women and 9 men) between the ages of 17 and 45 participated. A local ethics committee approved the experimental protocol. All subjects were right-handed and had normal vision or vision that was corrected for normal. Subjects were assigned to one of three groups. Subjects in the control group $(n=10)$ were presented with a visuomotor rotation session on successive days. Subjects in the visual-feedback group $(n=8)$ were presented with a visuomotor rotation session followed 5 min later by a force field learning session on day 1 ; they were then presented with the visuomotor rotation again on day 2 . The visuomotor transformation was turned off during adaptation to the force field. Subjects in the no-visualfeedback group $(n=8)$ were presented with the same sequence of tasks as subjects in the visual-feedback group, except that they did not receive visual feedback of the hand position during presentation of the force field. The control group contained 10 subjects because 2 subjects, originally scheduled for inclusion in another group, did not have time to complete two tasks on day 1 .

Apparatus. While seated, subjects grasped a lightweight, forcereflecting manipulandum (Phantom Haptic Interface 3.0; Sensable Technologies, Woburn, MA) that they moved to targets located in a horizontal plane. The targets and the position of the hand were represented as virtual spheres using a three-dimensional projection system with shutter glasses [for full details of the setup, see Goodbody and Wolpert (1998); subjects could not see their hand or arm (Fig. 1)]. The spheres representing targets (green) and the position of the hand (white) were $2 \mathrm{~cm}$ in diameter. The three-dimensional force exerted by the manipulandum on the hand was servo-controlled at $1 \mathrm{kHz}$ to create a position-dependent rotary force field in the horizontal plane (see below). Under the null force field, subjects experienced small forces associated with the passive mechanics of the manipulandum.

Procedure. Subjects made out-and-back movements to one of eight targets from a central starting position (or target) located $\sim 10 \mathrm{~cm}$ below shoulder level and in the subject's midsagittal plane. The targets were located radially about the starting position and in the same horizontal plane. All targets were located $15 \mathrm{~cm}$ from the starting position. Participants were instructed to move their hand out to the target and back to the starting position in a single, quick, continuous motion; they were asked not to make corrective adjustments during the movement. Under the visuomotor rotation, the position of the hand sphere was rotated $30^{\circ}$ counterclockwise about the starting position in the horizontal plane. Following the procedure used by Krakauer et al. (1999), targets were presented in a constant sequence, starting at $0^{\circ}$ (directly away from the subject) and continuing in positive, counterclockwise increments of $45^{\circ}$. A cycle was defined as eight successive trials from $0^{\circ}$ to $315^{\circ}$. Each session consisted of 30 cycles (i.e., 240 movements). To avoid fatigue, subjects were given a brief 1 min rest every five cycles (i.e., every 40 trials).

To begin each trial, subjects had to position the sphere representing their hands at the starting position. The trial was initiated only when the hand was within the starting area for a full second. At the start of the trial, one of the targets was presented and the subjects had to move out to the target and back to the starting position, which remained visible. To ensure that the participants were able to return to the starting position when visual feedback was not provided during the movement, we illuminated the cursor if the hand was off the starting location at the end of their out-and-back movement but within $5 \mathrm{~cm}$ of the starting location. Visual feedback of the position of the cursor was turned off at the start of the trials. To control movement time, a counter was started as soon as the hand moved $2 \mathrm{~cm}$ away from the starting position. After $450 \mathrm{msec}$, an auditory signal was provided and the starting position flashed red for 100 msec. The subjects' task was to arrive back at the starting position at the same time that these auditory and visual cues were turned on. Thus, the subject was encouraged to complete the out-and-back movement in 450 msec.

Transformations. Under the position-dependent visuomotor transformation, the position of the hand was rotated about the starting position (origin) in the horizontal plane using the following rotation matrix:

$$
\left[\begin{array}{l}
p \\
q
\end{array}\right]=\left[\begin{array}{cc}
\cos (\theta) & -\sin (\theta) \\
\sin (\theta) & \cos (\theta)
\end{array}\right]\left[\begin{array}{l}
x \\
y
\end{array}\right]
$$

where $x$ and $y$ are the coordinates of the hand in the horizontal plane relative to its starting location (Fig. 1 ), $\theta$ equals $30^{\circ}$, and $p$ and $q$ are the coordinates of the new, rotated "hand" position. Note that when the hand is located at the starting position $(x=0, y=0)$, the locations of the actual and visually perceived hand are identical.

Under the position-dependent rotary force field, the force applied to the hand by the manipulandum handle was proportional to the displacement of the hand away from the starting position and directed perpendicular to the hand displacement vector, such that the force tended to rotate the hand clockwise (in the direction opposite to the visuomotor rotation). The following equation was used to compute the force to be applied:

$$
\left[\begin{array}{c}
F_{\mathrm{x}} \\
F_{\mathrm{y}}
\end{array}\right]=k\left[\begin{array}{cc}
\cos (\theta) & -\sin (\theta) \\
\sin (\theta) & \cos (\theta)
\end{array}\right]\left[\begin{array}{l}
x \\
y
\end{array}\right]
$$

where $x$ and $y$ are the coordinates of the hand in the horizontal plane relative to the starting location, $F_{\mathrm{x}}$ and $F_{\mathrm{y}}$ are forces acting in the horizontal plane, $k$ equals $60 \mathrm{~N} / \mathrm{m}$, and $\theta$ equals $-90^{\circ}$.

Analysis. The three-dimensional position of the hand (center of the manipulandum handle) was recorded at $200 \mathrm{~Hz}$ using the encoders of the Phantom. To compute the velocity of the hand, these position data were first digitally filtered using a low-pass second-order Butterworth filter with a cutoff frequency of $14 \mathrm{~Hz}$. A three-point central difference equation was then applied to compute the velocities in three dimensions. The tangential velocity of the hand in the horizontal plane was computed as the resultant of the velocities in the $x$ and $y$ directions.

To quantify the learning of the position-dependent visuomotor rotation, we first computed movement direction defined as the instantaneous direction of cursor movement $150 \mathrm{msec}$ after the start of the movement. We then computed the angle between the direction of movement and the target direction (the direction of the vector from the start position to the target). We measured movement direction $150 \mathrm{msec}$ after the start of the movement so that our measure would not be affected by significant on-line corrections based on visual feedback of cursor position. The start of the movement was taken as the point at which the tangential velocity of the hand (in the horizontal plane) last exceeded $0.02 \mathrm{~m} / \mathrm{sec}$ before reaching a speed of $0.2 \mathrm{~m} / \mathrm{sec}$.

To quantify learning of the position-dependent rotary force field, we 


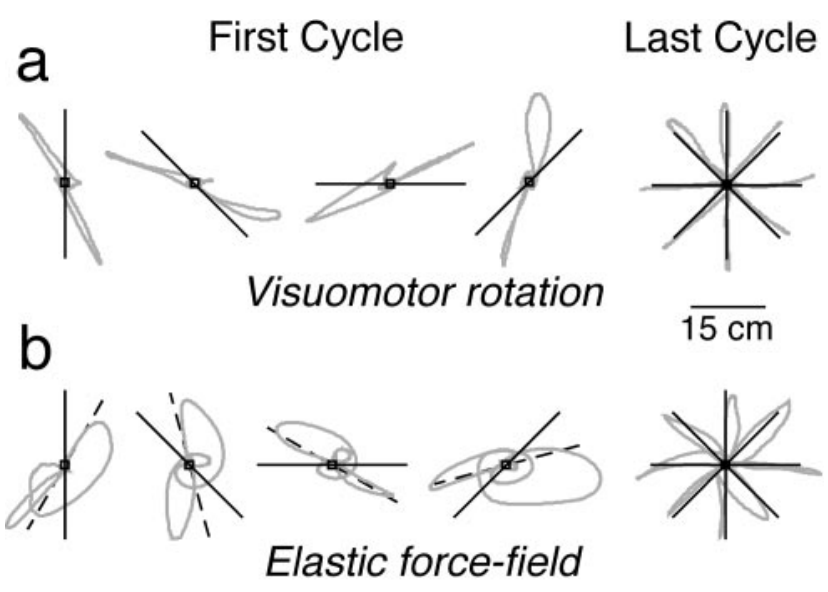

Figure 2. Performance in the first and last cycles under the visuomotor rotation $(a)$ and elastic force field with visual feedback $(b)$. The gray curves show the individual paths of the cursor representing hand position for a single subject (different subjects are shown in $a$ and $b$ ). The straight solid lines indicate the direction and extent of the corresponding targets. For the first cycle, two movement paths (directed to opposing targets) are shown in each plot. For the last cycle, all eight paths are shown in one plot. Because the force field was presented after adaptation to the visuomotor rotation, subjects initially directed their movements $30^{\circ}$ clockwise to the targets. These rotated directions are indicated by the straight dashed lines in $b$. In the first cycle, large errors were observed under both transformations. However, after 30 cycles, these errors were greatly reduced.

computed the normalized path length in the horizontal plane. Specifically, we divided the integrated path length by twice the maximum hand displacement from the starting position; in other words, we normalized the path length by the minimum possible path length given the displacement of the hand away from the starting position. This normalization procedure was used because, under the condition of no visual feedback, subjects could produce movements that either overshot or undershot the visual target. In such cases, the path length, without normalization, would be influenced by movement amplitude independently of path curvature.

\section{RESULTS}

\section{Hand paths at the start and end of adaptation}

Subjects in all three experimental groups were first exposed to the position-dependent visuomotor rotation, in which a cursor that represents the position of the hand was rotated $30^{\circ}$ from the position of the hand. Figure $2 a$ shows individual cursor paths to each of the eight targets for a single subject in the first and last (30th) cycles. As expected, in the first cycle, the paths were rotated counterclockwise with respect to the target direction (straight lines). However, after 30 cycles, the subject adapted and produced paths that were directed toward the targets.

After adapting to the position-dependent visuomotor rotation, subjects in two of the three groups were exposed to a positiondependent rotary force field, either with vision, in which case the visuomotor transformation was turned off, or without vision. Figure $2 b$ shows individual hand paths to each of the targets in the first and last cycles for a single subject in the visual-feedback group. Because the subjects had just adapted to the visuomotor rotation, they made direction errors in the first cycle. In particular, they directed their hand movements $30^{\circ}$ clockwise from the targets. (The targets are indicated by solid straight lines and the rotated targets are indicated by dashed lines.) In addition, in the first cycle, the rotary force field gave rise to large loops in the hand path. At the start of each movement, the hand paths were directed toward the rotated targets, but they then veered clock- wise as the hand was displaced from the origin and the rotary force increased. After 30 cycles, the subject once again adapted so as to produce relatively straight hand paths to the targets. This involved both an unlearning of the visuomotor rotation and an adaptation to the rotary force field.

\section{Interference between kinematic and dynamic learning}

Figure 3 shows learning curves under the visuomotor rotation on day 1 (solid curves) and day 2 (dashed curves) for each of the three experimental groups. To construct these curves, we first computed the mean directional error for each subject and cycle (averaging across the eight movements within each cycle) and then computed the average directional error across subjects for each cycle. The height of the gray area represents $1 \mathrm{SE}$. Figure $3 a$ shows the results for the control group subjects, who were exposed to the $30^{\circ}$ visuomotor rotation on day 1 and then retested on day 2 . On day 1 , the subjects initially produced directional errors close to $30^{\circ}$. After 30 cycles (or 240 movements), their errors were greatly reduced. When the same subjects were retested $24 \mathrm{hr}$ later, the initial directional errors were much smaller, indicating substantial retention of learning. Note that even at the end of day 2 there was a small directional bias of $\sim 5^{\circ}$, which is consistent with previous reports (Krakauer et al., 1999; Wigmore et al., 2001).

Figure $3, b$ and $c$, shows the results for the two groups of subjects exposed to the position-dependent force field 5 min after adapting to the position-dependent visuomotor rotation on day 1 . The data shown in Figure $3 b$ are for the group who received visual feedback of the cursor position during adaptation to the force field; the data in Figure $3 c$ are for the group who did not receive visual feedback. There was a small improvement in performance from day 1 to day 2 in both groups. However, in comparison with the control group (Fig. $3 a$ ), it is apparent that adapting to the force field on day 1 interfered dramatically with the retention of the visuomotor rotation that would otherwise be expected.

In all of the curves shown in Figure 3, small peaks can be observed every five cycles. These are the cycles that followed the 2 min rest breaks. Although the rest breaks resulted in transient increases in direction error for a single cycle, these increases did not appear to affect the shape of the learning curve otherwise. Note that the decrease in direction error from the cycle immediately after the break (i.e., the cycle exhibiting the peak in error) to the next cycle tended to be large. Thus, qualitatively, subjects appeared to recover rapidly after the break and stay on course in terms of adaptation.

To quantify the learning curves shown in Figure 3, we adopted the procedure used by Krakauer et al. (1999). Specifically, for each subject we computed the average directional error over the second and third cycles as well as the average directional error over the last two cycles (cycles 29 and 30). We then averaged these errors across subjects to obtain mean values. The bar graphs in Figure 4 depict the mean directional errors over the second and third cycles for each experimental group and for day 1 (solid bars) and day 2 (dashed bars). The inner gray bars represent the corresponding directional errors over the last two cycles.

To test our main hypotheses, we performed two comparisons involving orthogonal partial interactions between day and group with the directional error over the second and third cycles as the dependent variable. First, to assess the overall effect of dynamic learning (independent of visual feedback conditions) we examined the interaction between day and group while combining 


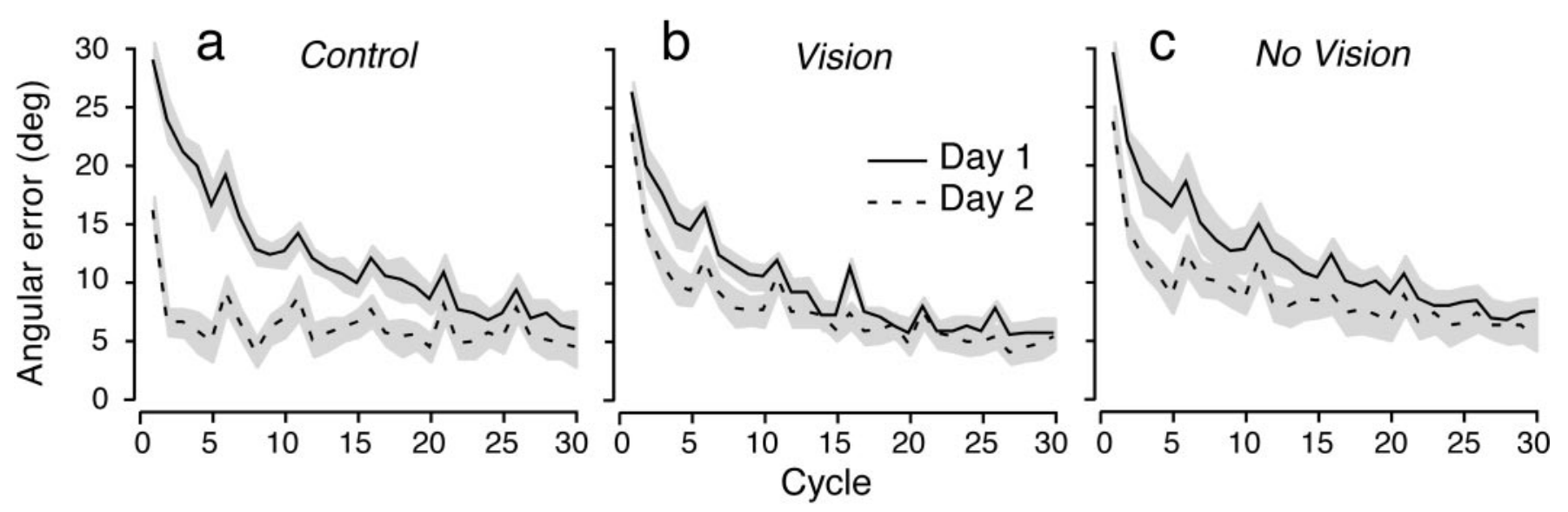

Figure 3. Adaptation to the visuomotor rotation. Curves show mean angular error (between movement and target directions) as a function of cycle on day 1 (solid lines) and day 2 (dashed lines). The height of the gray area represents \pm 1 SE. $a$ shows results for the control group, who adapted only to the visuomotor rotation on day $1 . b$ and $c$ show results from two groups, who on day 1 adapted to the visuomotor rotation and 5 min later adapted to the position-dependent rotary force field either with $(a)$ or without $(b)$ visual feedback. The two groups exposed to the force field on day 1 exhibited greater directional errors at the start of day 2 than did the control group.

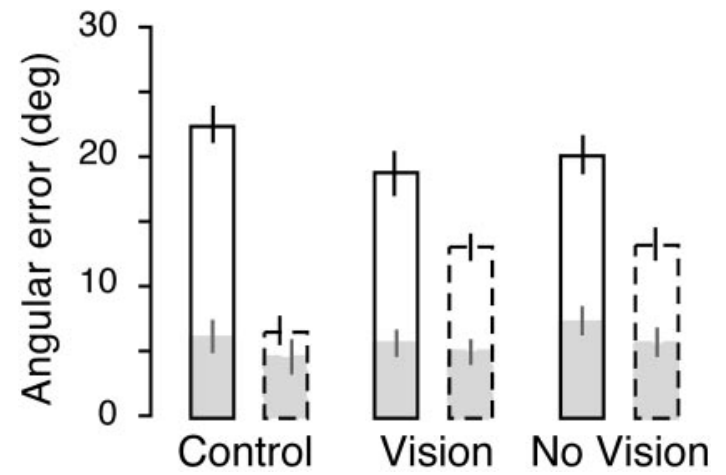

Figure 4. Initial and final angular errors under the visuomotor rotation. The height of each white bar represents the angular error averaged over the second and third cycles; the height of each gray bar represents the corresponding angular error averaged over the 29th and 30th cycles. The bars outlined with solid and dashed lines represent days 1 and 2, respectively. Separate means are reported for each of the three experimental groups. Vertical lines represent the SE.

groups 2 and 3. A highly reliable effect was observed $\left(F_{(1,24)}=\right.$ 26.76; $p<0.001)$, indicating that dynamic learning interfered with kinematic learning. To test whether this effect was mediated by visual feedback conditions, we assessed the orthogonal interaction between day and group in which only groups 2 and 3 were included in the analysis. In this case, a significant interaction was not found $\left(F_{(1,16)}=0.41 ; p=0.531\right)$. Thus, we found no evidence that the level of interference observed between dynamic and kinematic learning depends on whether subjects received visual feedback during dynamic learning.

Exposure to the position-dependent rotary force field clearly interfered with retention of the position-dependent visuomotor rotation. However, limited retention of the latter was observed. When combining the two groups exposed to the force field (groups 2 and 3), the initial directional error (in the second and third cycles) was $19.4^{\circ}$ on day 1 and $13.2^{\circ}$ on day 2 , an improvement of $6.2^{\circ}$. Although this level of retention was markedly smaller than the level observed for the control group (a difference across days of $\left.15.8^{\circ}\right)$, it was nevertheless reliable $\left(F_{(1,16)}=38.41\right.$; $p<0.001)$. Thus, dynamic learning did not result in a complete absence of consolidation of kinematic learning.

\section{Adaptation to the position-dependent force field}

Our finding that exposure to the position-dependent rotary force field interferes with kinematic learning suggests that subjects did in fact adapt to and learn the force field. Figure 5 shows that this was indeed the case. The curves in Figure $5 a$ show normalized path length (a measure of the curvature of the hand path) as a function of the cycle for both the visual-feedback group (dashed line) and the no-visual-feedback group (solid line). Each point on the curve represents the mean of subject means, each averaged across the eight movements within a cycle. In both groups of subjects, the normalized path length decreased markedly over the first 10 cycles or so and then decreased more gradually thereafter. After 30 cycles, the normalized path length approached 1.1 for both groups; a perfectly straight path out to and back from the target would result in a normalized path length of 1 .

While adapting to the position-dependent rotary force field, subjects also unlearned the visuomotor rotation they learned before they were exposed to the force field. This "de-adaptation" is evident in Figure $5 b$, which shows mean directional error (averaged across subjects and based on subject means) as a function of cycle. As can be appreciated visually, the rate of de-adaptation (or re-adaptation to the $0^{\circ}$ rotation) was slightly faster when visual feedback of the cursor position was provided. Moreover, the absolute directional error after 30 cycles was slightly smaller in the visual-feedback group. However, the differences between the two curves are small, and it is striking how quickly subjects in the no-visual-feedback group unlearned the visuomotor rotation, presumably by comparing visual information about the target with proprioceptive information related to arm position.

\section{DISCUSSION}

We have demonstrated clear interference between kinematic and dynamic learning. First, we showed that when subjects adapted to a position-dependent visuomotor rotation and were tested on the same visuomotor rotation a day later, their performance was greatly improved. This result is consistent with previous studies (Krakauer et al., 1999; Wigmore et al., 2001); it indicates that the knowledge of the visuomotor rotation was consolidated in longterm memory. Second, we demonstrated that when subjects successively adapted to a position-dependent visuomotor rotation 

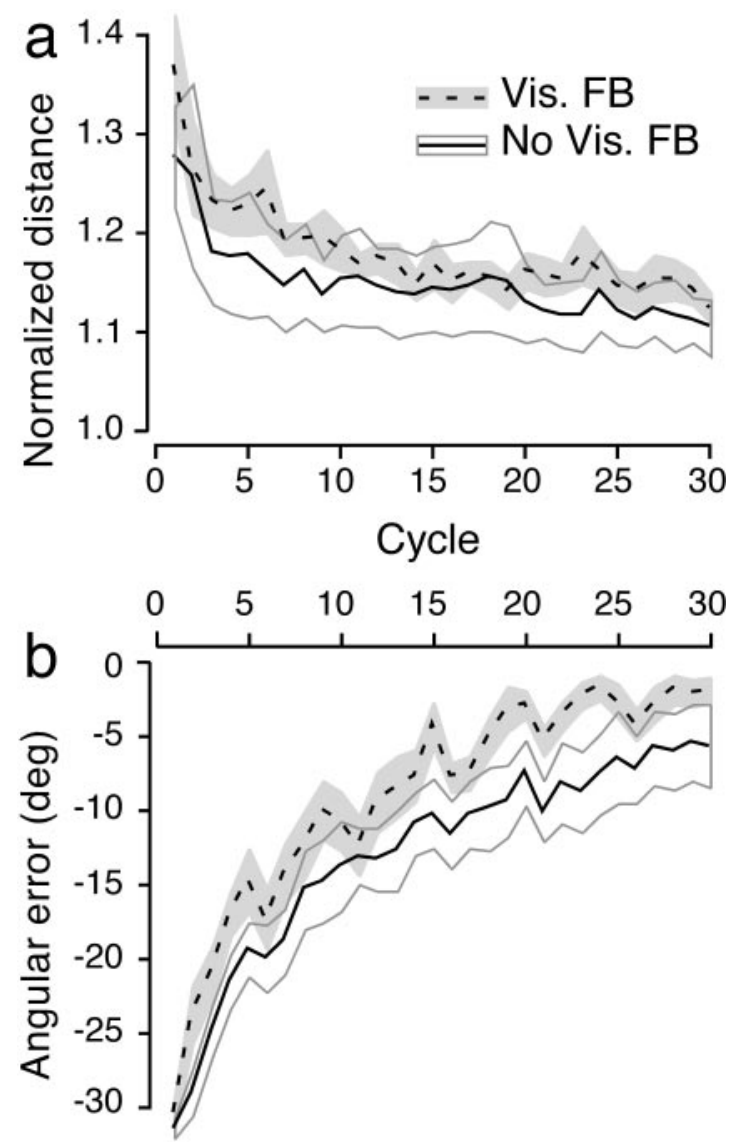

Figure 5. Adaptation to the elastic rotary force field. $a$, Distance traveled by the hand as a function of cycle (normalized for maximum displacement of the hand). The dashed and solid lines represent mean distances for the visual feedback and no-visual-feedback groups, respectively. The height of the gray area around the dashed curve and the gray line around the solid curve represent \pm 1 SE. $b$, Corresponding mean angular errors for the two groups. For both groups, the magnitude of the angular error decreased as the visuomotor rotation previously adapted to was gradually unlearned during adaptation to the force field.

and then to a position-dependent rotary force field, retention of the visuomotor rotation was significantly impaired when performance was tested the next day. This retrograde interference indicates that learning and consolidation of the visuomotor rotation was disrupted by adaptation to the rotary force field.

Previous work has demonstrated retrograde interference between opposing kinematic (Krakauer et al., 1999) and opposing dynamic (Brashers-Krug et al., 1996) sensorimotor transformations that are presented close together in time. Thus, when subjects adapt to two opposing visuomotor rotations presented 5 min apart, there is no improvement in performance on the first rotation when tested a day later (Krakauer et al., 1999). Similarly, when subjects adapt to a velocity-dependent force field that generates rotary forces in one direction and then adapt $5 \mathrm{~min}$ later to a field that generates rotary forces in the opposite direction, performance on the first force field is not improved when evaluated the next day (Brashers-Krug et al., 1996). In contrast, Brashers-Krug et al. (1996) have shown that when the two opposing force fields are presented $\sim 6 \mathrm{hr}$ apart, retrograde interference is no longer observed. That is, subjects exhibit normal retention on the first force field when retested a day later. These authors also found that anterograde interference (i.e., a deficit in performance on the second force field attributable to prior learning of the first) decreased over this period [but see Bock et al. (2001) for an example of long-lasting anterograde interference]. Based on these results, Brashers-Krug et al. (1996) argued that motor learning involves a two-step process in which knowledge acquired during practice is initially stored as an internal model in working memory and then gradually consolidated in long-term memory. The internal model in working memory is fragile and can be interfered with by new learning that competes for the same resources. In contrast, once consolidated in long-term memory, the internal model is stable and is not susceptible to retrograde interference by new learning (Brashers-Krug et al., 1996; Shadmehr and Brashers-Krug, 1997). The results presented here indicate that position-dependent and rotational kinematic and dynamic transformations compete for common resources in motor working memory and argue against the idea that separate working-memory systems underlie kinematic and dynamic learning (Krakauer et al., 1999).

Our results appear to be in direct disagreement with those of Krakauer et al. (1999), who found independent learning of kinematic and dynamic transformations. They showed that when subjects successively adapt to a visuomotor rotation and then to an inertial load, adaptation to the inertial load does not interfere with learning and retention of the visuomotor rotation. We believe that the difference between the two sets of results is best explained in terms of the nature of the kinematic and dynamic transformations used. Krakauer et al. (1999) used transformations that depended on different kinematic parameters, namely a position-dependent visuomotor rotation and an inertial or acceleration-dependent load. In contrast, we used two transformations that were equal in terms of the kinematic parameters on which they depended. Thus, our results suggest that separate motor working-memory resources may be used when learning transformations that depend on different kinematic parameters. Note that this hypothesis is not jeopardized by the results of Flanagan et al. (1999), who found that there is no transfer of learning when subjects successively adapt to a position-dependent visuomotor rotation and a velocity-dependent rotary force field. Although this lack of transfer supports the idea that kinematic and dynamic learning are independent, the transformations involved depended on different kinematic parameters.

Although we have argued that the key difference between our study and the study by Krakauer et al. (1999) is whether the transformations involved depend on the same or different kinematic parameters, there are several other differences between the two studies that may have contributed to the difference in results. In our experiment, forces under the dynamic transformation were applied through the hand, whereas in the study by Krakauer et al. (1999), forces were applied via a support attached to the forearm. It might be argued that the dynamic transformation used by Krakauer et al. (1999) is learned in intrinsic coordinates related to the sensors and muscles of the arm, whereas the dynamic transformation we used is learned in extrinsic coordinates related to the position of the hand in space. Because visuomotor rotations appear to be learned in extrinsic coordinates (Krakauer et al., 2000), the difference between our results and those obtained by Krakauer et al. (1999) may relate to the coordinate systems in which the transformations are learned. However, previous work on motor adaptation to dynamic perturbations indicates that force fields applied to the hand are represented in intrinsic coordinates (Shadmehr and Mussa-Ivaldi, 1994; Gandolfo et al., 1996). 
Another important difference between our study and that of Krakauer et al. (1999) concerns the properties of the sensory error signals and motor adjustments brought about by the kinematic and dynamic transformations. The two transformations used in our study produced rotary errors in opposite directions and, likewise, required opposing motor adjustments. Under the visuomotor rotation, subjects had to generate commands that would send their arm to the right of the visual target, whereas under the elastic force field, they had to generate commands that would send their arm to the left of the visual target. In contrast, the dynamic perturbation used by Krakauer et al. (1999) did not appear to rotate the position of the hand away from the target significantly. Thus, the kinematic and dynamic transformations produced sensory errors and required motor adjustments that were neither both rotational nor both opposite. It may be that the interference we observed between kinematic and dynamic learning is related to the fact that our transformations required somewhat opposing motor adjustments. Indeed, previous work has shown that two kinematic tasks that depend on the same dependent variable interfere with one another only when the transformations involve conflict (Bock et al., 2001).

We observed statistically equivalent interference effects regardless of whether or not subjects received visual feedback of the cursor position while adapting to the rotary force field. Thus, the interference we observed between kinematic and dynamic learning does not depend on receiving sensory feedback in the same (visual) modality. Moreover, receiving somewhat opposing visual feedback when adapting to the kinematic and dynamic transformations does not increase the magnitude of the interference. To the extent that opposing sensory errors might be responsible for the observed interference, our results suggest that these errors may be represented in a common coordinate system in motor working memory.

In summary, we have shown that kinematic and dynamic transformations are not learned independently when the transformations are both rotary and position-dependent. We hypothesize that the kinematic parameter on which a transformation depends is a key factor mediating the allocation of resources in motor working memory. Thus, if two transformations depend on the same kinematic parameter and also require opposing sensorimotor adjustments, they should interfere with one another. The strong prediction from this hypothesis is that two dynamic or two kinematic transformations should not interfere with one another if they depend on different kinematic parameters.

\section{REFERENCES}

Bock O, Schneider S, Bloomberg J (2001) Conditions for interference versus facilitation during sequential sensorimotor adaptation. Exp Brain Res 138:359-365.

Brashers-Krug T, Shadmehr R, Bizzi E (1996) Consolidation in human motor memory. Nature 382:252-255.

Conditt MA, Gandolfo F, Mussa-Ivaldi FA (1997) The motor system does not learn the dynamics of the arm by rote memorization of past experience. J Neurophysiol 78:554-560.

Flanagan JR, Wing AM (1997) The role of internal models in motor planning and control: evidence from grip force adjustments during movements of hand-held loads. J Neurosci 17:1519-1528.

Flanagan JR, Nakano E, Imamizu H, Osu R, Yoshioka T, Kawato M (1999) Composition and decomposition of internal models in motor learning under altered kinematic and dynamic environments. J Neurosci 19:RC34:1-5.

Gandolfo F, Mussa-Ivaldi FA, Bizzi E (1996) Motor learning by field approximation. Proc Natl Acad Sci USA 93:3843-3846.

Goodbody SJ, Wolpert DM (1998) Temporal and amplitude generalization in motor learning. J Neurophysiol 79:1825-1838.

Johansson RS, Cole KJ (1992) Sensory-motor coordination during grasping and manipulative actions. Curr Opin Neurobiol 2:815-823.

Kawato M (1999) Internal models for motor control and trajectory planning. Curr Opin Neurobiol 9:718-727.

Krakauer JW, Ghilardi MF, Ghez C (1999) Independent learning of internal models for kinematic and dynamic control of reaching. Nat Neurosci 2:1026-1031.

Krakauer JW, Pine ZM, Ghilardi M-F, Ghez C (2000) Learning of visuomotor transformations for vectorial planning of reaching trajectories. J Neurosci 20:8916-8924.

Miall RC, Weir DJ, Wolpert DM, Stein JF (1993) Is the cerebellum a Smith Predictor? J Motor Behav 25:203-216.

Shadmehr R, Brashers-Krug T (1997) Functional stages in the formation of human long-term motor memory. J Neurosci 17:409-419.

Shadmehr R, Mussa-Ivaldi FA (1994) Adaptive representation of dynamics during learning of a motor task. J Neurosci 14:3208-3224.

Wigmore V, Tong C, Flanagan JR (2001) Visuomotor rotations of varying size and direction compete for a single internal model in motor working memory. J Exp Psychol Hum Percept Perform, in press.

Wolpert DM, Ghahramani Z (2000) Computational principles of movement neuroscience. Nat Neurosci 3:1212-1217.

Wolpert DM, Ghahramani Z, Jordan MI (1995) An internal model for sensorimotor integration. Science 269:1880-1882. 